\title{
Analysis on the Current Situation of the Development of Chibi's Primary and Secondary School Football
}

\author{
Gen LI
}

Wuhan Business University, 816 Dongfeng Road, Wuhan City, PRC

Keywords: Campus football, Physical education teacher, Economic base.

\begin{abstract}
The main contradiction faced by Chinese football is the contradiction between the growing material and cultural demand of the people and the development of the football. To resolve this major contradiction, you must start with a "soccer ball from the doll". The youth is the future of the country, the foundation of national strength, the hope of realizing the revitalization of sports, and the expectation of all sports workers. "Campus football" is a great innovative practice to reform China's traditional football reserve talent training system. Education's development strategy combined with the sports department has provided realistic possibilities for the realization of our country's independent training of high quality and high level football talents.

Confucius wrote in the book of prequel, "the poor will change, the other will change, the general will be long". Sports administration, the ministry of education jointly issued by the notice about the national youth campus football activities for China football a good recipe was made out of fall, also for those in the Chinese football still to pursue his dream of football in the winter the children sent to a fire of coals. The campus football activities carried out in China is not only beneficial to expand the population, consummates our country football reserve talent cultivation system, can improve physical quality level of adolescent students in our country, which lay a foundation for the full implementation of quality education policy.

This paper aims to chibi hubei teenagers of primary and secondary schools to carry out the investigation and analysis of the present situation of the campus football activities, find chibi hubei school football teenagers do appear in the process of problem, existence of the problem analysis, for the future campus football in chibi, hubei province and even the development of small and medium-sized cities in hubei province to provide a valuable material and reference.

This paper mainly analyzes the current situation of school football in junior and middle schools in chibi city by means of literature, investigation and mathematical statistics. The questionnaire is divided into four categories: PE teacher (coach) questionnaire, student questionnaire, school leadership questionnaire and parent questionnaire. Through the analysis of effective recycling questionnaire, chibi to a comprehensive understanding of the present situation and problems of primary and middle school campus football teenagers, chibi expect schools found that football in the universality of conditionality factors exist in the process.
\end{abstract}

Question

(1) the development of school football activities in primary and secondary schools in chibi city is facing: the football field is not standardized; The football teacher's degree and professional title make up more reasonable but the football professional skill needs to be improved; Most schools use extracurricular activities or soccer practice, and fewer schools offer football courses.

(2) the problems of physical education teachers in the main body of activities include: low job satisfaction; Salary is not proportional to the amount of work; There is a lot of pressure to organize and carry out football activities.

(3) the most prominent problem encountered by students participating in the activity is that there are not many competing opportunities and there are fewer students in the competition.

(4) the main concerns of the school leaders of activities management are the problems of students' safety and the maintenance of school teaching order. 
(5) parents of students against their children's participation in football activities mainly worry that their children's participation in football activities will affect their children's academic performance. Besides, they fear that their children will be hurt by sports.

Countermeasure

(1) increase financial support for school football and improve the construction of football fields and facilities in primary and secondary schools; Widen the channel of teaching ability of football teachers and increase the introduction of high level talents; To encourage primary and secondary schools to develop soccer school curriculum and form a football curriculum system with the characteristics of the school

(2) improve the teaching status of football teachers; The daily training and competition will be included in the total amount of teaching work of football teachers, and improve the salary treatment of football teachers. We will intensify efforts to support the work of football teachers and ensure the smooth development of their work.

(3) make full use of the advantage resources of the administrative functional departments and develop social resources to increase the opportunities for primary and middle school students to participate in football related activities.

(4) enrich the insurance system for students to participate in activities, strengthen the safety education of student participation activities, and make detailed activities safety plan to minimize the risk of injury to students;

(5) pay attention to social propaganda work, carry forward the positive energy of football, maintain a good image of football, and improve public recognition of the positive function of football from the social aspect.

\section{Introduction}

Football has enjoyed the world's largest sports in the world, a high level of international football matches its influence even beyond the game itself, not only can stimulate the enthusiasm of millions of fans and create great economic benefits at the same time, as an important form of international cultural exchange, the countries in the world of football can improve and strengthen between the country and the country, so as to promote the harmonious development between the world within the scope of the main body of the.

All levels of the national team dismal results the result should not only be for the masses to quell and Voices of discontent. Dismissal of coach, expelled from the individual players, but also should be given China football people have a firm, a firm that is to combine the campus football body to teach Road, change the selection of our country all levels of football talents with training methods, will achieve the football dream and realize Chinese dream together, for the continuous efforts. The main contradiction in Chinese football is the contradiction between the demand of material and culture and the level of football development. The fundamental way to solve this contradiction is "football should be picked up from the doll." A person at a young age, psychology and physiology are in the developmental stage, has great plasticity, easy to accept and learn new things, as the future of the country and national power storage, young people entrusted with the revitalization of the country sports hope, also carrying the great expectations of the sports workers. The rise and rise of "campus football" has revolutionized the traditional mode of training football talents in China, which is a huge and innovative practice [1]. The development strategy of the education department is integrated with the sports department, which provides a realistic possibility for the self-cultivation of high-quality football talents.

As we all know, football is good or bad in the development of a country. The key factor of influence is the reserve of domestic football professionals. In the process of rapid economic and social development in China, it is bound to cause great changes in all aspects of society. Coupled with the lagging characteristics of relevant legal system and regulatory system, it inevitably leads to chaos in a certain industry in a certain period. After the occupation occupation League, football clubs in China tend to pursue short-term sensational effects while ignoring its sustainable development, 
training, reserve personnel echelon construction system is extremely imperfect, occupation football club can hardly bear the important task of football talents, caused by the loss and lack of ability, so that the development of football in China by to a great extent hinder.

In January 22, 2014 tenth People's Republic of China Football Conference, the new generation will put the "Chinese foot football long-term planning outline", it is pointed out that the development of our country's football focus is to carry out the campus football activities to increase the football population, further improve the training of reserve football. Through the nationwide "campus football" activities, in order to enable the majority of students to recognize football, experience football and feel football, the football is introduced into the campus, and then form a distinctive campus football culture. The activities of "campus football" have been carried out rapidly throughout the country, and the research on the problem of campus football has gradually increased.

\section{Research Status}

\section{Domestic Research Status}

Through the retrieval of "HowNet", "China WanFangDATA" (2015-2017) database, to the "campus football", "Hubei campus football" and "school football" as key words, search related to football in development of primary and secondary literature every year there are more than 50 articles. Based on the related literature retrieval to read, for the study of youth football in our province are mainly concentrated in the study of football talents training system, and macro research and theoretical research, the research for today's training mode is relatively less in China Youth talent. For primary and secondary schools to carry out research on football mainly for Beijing, Shanghai, Guangzhou, on Wuhan, Liaoning and other economically developed areas or traditional football school football activities; in the research on the campus football activities in a province and city as the research scope, in the face of a specifically, the campus football activities carried out in the problem put forward the improvement suggestions, in county-level administrative units are rare as the object of study on campus football activities.

Guan Li Zhang Hongjia, starting from the perspective of family factors in the investigation and analysis of "family" factors affecting youth football popularity of a text, in order to carry out the problem with the traditional football city primary school campus football activities as the main research direction and breakthrough in the past will make main positions for amateur training of juvenile football talent thinking the main battlefield of the primary school, the school of football amateur training "as the research group of youth football, with the family as the main consideration of students participating in football activities factors and breakthrough. Based on the analysis of the basic situation of primary school students in the urban area of Shenyang, this paper discusses and understands the family factors that may affect the development of youth soccer in Shenyang from the whole family level of school football training students. [2]

Liu Bing in the "present situation and development countermeasure research" at this stage of our school football amateur training which, since the occupation change from a global perspective on the overall situation of system, launched football training in the campus and the problem of the comprehensive discussion of China today launched the football amateur training activities on campus the present situation and the restriction factors of the reason analysis, and puts forward several fit our school football training practice recommendations, including: the school football training work should adhere to the core content of personnel training; school football training must keep the organic connection of training system and education system of the principle of welfare distribution; attention in school the football amateur training of all forces work [3].

Zhou Zhicheng mentioned in the "investigation" of middle and primary schools in Wuhan women's football in the Wuhan area: the expansion of primary and secondary schools to participate in sports activities of the women's football amateur training or rely on their strength to support women's teams of two, ignoring the important function of mutual integration of women's football sport and education two Department of resources, and to ignore the use of talents and training full-time schools to 
mobilize resources; make full use of human resources and the site of the local primary and secondary school, the school can play in the women's football athlete selection, forming a huge advantage and set up the daily training and teaching in life; to ensure that in the full-time school in primary and secondary expansion team the project takes place, the Football Association for local schools to expand grassroots coaches training channels; Wuhan City Many primary and secondary schools generally have problems of low effective utilization of football venues. In addition, the development of women's football in Wuhan is also facing the problems that the National Women's football is facing generally, that is, the treatment of the active members is low, and the employment difficulties of retired players are [4]. According to the present situation of the proposed measures include: "emancipating the mind" at home and abroad to learn the advanced experience of women's development, the sports sector and the education sector need to cooperate closely, make full use of the full-time schools good resources to promote the development of the women's movement; full development of human resources in the primary and secondary schools, and actively create into soccer movement opportunities in sports activities of girls primary and secondary schools, sports experience the charm of soccer; the relevant functional departments work together to increase the development of women's sports funding and policy support efforts to improve women's athletes to be met, solve the retired after learning and work, to maximize the elimination of girls participating in football concerns; finally, we should give full play to the sports department of professional and technical advantages development, teachers of primary and middle school teachers or football training channels, improve primary and secondary schools The professional skills of a football coach [5].

Liu Weimin "Wuhan city youth soccer movement development main factor analysis and Countermeasures of" the restriction of Wuhan urban primary and secondary schools on campus football activities of the various factors, put forward in the school football management system: the premise in the full play of Wuhan City Football Association of professional advantages, to mobilize the enthusiasm of sports school teachers, students and parents, grassroots leaders and ordinary students to participate in the football activities. The main problems existing in primary and secondary schools in Wuhan and for the teachers, venue, football culture knowledge are analyzed, and put forward the improvement strategy actively, so as to better promote the youth soccer movement in Wuhan city to carry out this insight [6].

To sum up, the problems facing the development of primary and secondary schools in our province today are complex and diverse, but the concentration is not high.

\section{Research Status in Foreign Countries}

In today's football development in more developed countries, generally will be placed in the campus football treat their football based strategic position, and gradually clear from a big trend, which combines the advantages of school resources development of football is the foundation to ensure the stable and healthy development of national football sports level.

The Republic of Korea. South Korean football talent development in the contemporary culture through the following two main modes: one is the system of training from primary school to University of the Dragon school football reserve personnel training system; the other is the youth football youth football club, classroom, occupation club as the main force of the club football talents. By the education department sports revitalization Bureau overall plan, the school sports department specific implementation school soccer reserve personnel training system in South Korean soccer talent training mode is in the main position [7]. The growth of Korean Teenager footballers usually needs three stages: primary, secondary and university. Therefore, it is not difficult to explain [8] in Korean high school football league and college football league level. Besides, the talent training system of Korean professional league clubs and immature football clubs is an important complement to the training mode of school football talents. In addition, the Korean Football Association has not regularly organized the domestic 14-16 year old players to the South American and European excellent clubs to further further the [9]. In the end, the Korean Football Association also paid 
considerable attention to the selection of young football talents. It is not difficult to see that campus football has been paid great attention to and paid attention to in Korea.

Germany. German football has been in the leading position in the world football world for many years, and its football training has always been a hot spot in world football research [10]. German football is largely dependent on its advanced talent training and scientific talent training system [11]. Germany began to implement a new reserve mode from 2000: 6 - 10 years old children in primary schools organize and with the popularity of football is the main purpose of the "campus football activities"; 11 - 18 year olds showed certain football talent and competitive level of football talent, can be added to the 338 a football base all over Germany itself, these funds are the base run by the German Football Association burden, the purpose is to make the best young football talent to get high level training opportunities for [12]. Players who perform well in football base training have the chance to enter the youth training camp organized by professional football clubs. The operation funds of the training camp are fully responsible for the club, and players will enter the club after the training camp. This comprehensive selection and training system integrating school football, national training base and professional football club can integrate all levels of advantageous resources, and ensure the scientific and continuous [13] of selection and training of reserve talents. In addition, schools can cultivate high level players and get reputation. Clubs can get youth who have potential to become high level football players, whether schools or clubs can get benefits from this system, so all parties will actively participate in the system.

This study through the overview of youth football advanced countries to carry out campus football, experiences from the campus football advanced city of our country, in the previous primary and junior high schools in Hubei Province youth football activities process characteristics at the same time, investigating the status quo of football in primary and middle schools in Hubei city of Chibi Province on the campus of youth, universal primary and secondary schools in Chibi city to carry out the activities of exposed in the process were analyzed, and provide valuable reference for decision-making and management department, Hubei city of Chibi province to expect the campus football from the theory to the practice of a leap.

\section{Research Objects and Methods}

\section{Research Object}

This paper is based on the current situation of school football in primary and secondary schools in Chibi, Hubei. The subjects of the survey include physical education teachers (instructors and coaches), students, parents of students, and related leaders and leaders.

\section{Research Method}

Document Method. With reference to the research needs, access to the Wuhan business school library collections and digital resources, integrated a variety of cyber source such as CNKI, Sina, Tencent, the campus football, youth football training, football talent training mode for the keyword index literature on campus football more than 300 articles carried out in primary and secondary schools football activities of more than 200 articles, youth football teaching more than 100 articles on youth training more than 150 articles, to lay the theoretical foundation for research.

Questionnaire Survey. Through a questionnaire survey, we have a comprehensive understanding of the development status of Chibi youth campus football activities, and provide reliable data and factual basis for our research and analysis.

The Formulation of the Questionnaire. Reference to the design of research and writing direction, for discussion by experts on the related problems, are designed in the football teachers students and parents, school leaders (coaches), these four kinds of problems related to the questionnaire, through detailed access to all kinds of school football main people, thus draw the conclusion to carry out detailed football problems the school of Chibi city. 
The reliability test of the questionnaire relied on the retest method. Within a month after the first questionnaire was recovered, $30 \%$ of the total number of the returned questionnaires was randomly selected to do the investigation again to analyze whether the results meet the requirements and ensure the reliability of the recovered questionnaires.

According to the total number of students in grade activities in schools, questionnaires were distributed according to the proportion, and the questionnaires were distributed evenly in each grade. There were nine grades in each grade.

Teacher questionnaire: only one teacher who is mainly responsible for the teaching or training of football in the school.

Students' parents questionnaire: in accordance with all the students participating in the questionnaire survey, $10 \%$ of the total number of participants were issued by the parents questionnaire.

School Leadership Questionnaire: the issuance of the leadership questionnaire is distributed according to the principle of one part of the school.

Release and recovery: form is mainly applied to send and receive the form, taking into account the low grade students' reading ability is limited by the class teacher preaching way, help the low grade students completed the survey participation.

Mathematical Statistics. Using sports statistics, mathematical methods and Excel software for data analysis, sorting, and using chart form to reflect the results of the survey.

Logical Analysis. From the questionnaires in the analysis of soccer school in Chibi city to carry out the actual situation faced in the process and common problems, and combining with our country and our province other city school lessons learned from the experience of the implementation of football activities in the process of construction of the primary and secondary school football system of Chibi and the status of the general issues in-depth inquiry.

\section{Results and Analysis}

\section{The Present Situation of Football Development in Primary and Secondary Schools in Chibi}

Site Facilities. Sports venues are the basic guarantee for school sports, and high quality school football field is an important factor to stimulate students to participate in the interest of football.

From table 1 , it is concluded that $16.7 \%$ of the primary and secondary schools in Chibi have standard soccer fields, $33.3 \%$ of the school grounds are 300 meters, 33.3\% of the school sites are 200 meters, and $16.7 \%$ of the school sites are 150 meters. So it can be seen that more than $80 \%$ of the primary and secondary schools in Chibi are irregular sites (below 300m). In addition, in the investigation of the site material, we know that there are only a small number of plastic fields and artificial grass fields in the investigated schools, and most of the school football fields are soil courts. Kicking a ball on a hard earth ground or a plastic field on the ground is much more likely to be injured than artificial meadows and natural meadows. From the field investigation, we can find that there are generally insufficient standards in Chibi primary and secondary schools, and the conditions of the venues are not ideal. This is one of the main restrictive factors that affect the development of primary and secondary school football. The lack of hardware facilities seriously restricts the development of football in primary and secondary schools. On the hardware facilities to improve and repair becomes critical, only increase in football related infrastructure investment, especially the facilities, let more students in sports standards and safety on site, in order to stimulate students' interest in football, promote the development of the campus football sports.

Educational Background of Football Teachers. The survey data on the degree of football teachers, in all subjects, the football school teachers with a bachelor's degree is 15, the proportion is $50 \%$, the proportion of teachers with college degree is $33.3 \%$, or 10 ; specialist qualifications of teachers for 5 people, accounted for only $16.7 \%$ of the total. The secondary school football teachers in Chibi have a high level of education. But after investigation, it is found that among the football 
teachers surveyed, most of the non football majors graduated, and the number of teachers who graduated from football majors or had professional football experience was relatively small. Most of the sports teachers' experience in teaching football comes from the training of the football coaches accepted after teaching. The lack of experience and practice with football football professional skills of teachers is to carry out the weak links of football in primary and secondary schools in Chibi City, the most elementary and high school football games and football teachers lack training experience in professional football; learning skills and training, the majority only rely on the campus football activities in the training of coaches in the channel; they in football professional skills and practice of football teaching and training to carry out the operation, especially for young football talents and development of universal law and differences in cognition have many shortcomings. The problems reflected in teaching activities and training process shows, is part of the school teachers can not guarantee the correct demonstration of the action research on youth training theory is not enough, as the sports team coaches coaching ability not people compliment, lack of practice ability school football teaching has become a major bottleneck in our province football academy work. The reason: professional football athletes in daily life in training professional skills training for the center of life, often can not guarantee the acceptance of cultural course of formal education, physical propagation and daily training and competition tasks consume huge makes these players no more energy to continue to study in training I. In the face of the most basic educational requirements for primary and secondary school teachers, retired football players are often unable to meet. The high level retired athletes are unable to teach, and it is a common problem in schools in all places.

An Investigation and Analysis of Football Classes of Football Teachers in Various Pilot Schools. An important factor for evaluating the professional quality of football teachers is the level of football, which affects the teacher's ability in teaching football to a certain extent. Table 3 shows that: There are no national footballers in 30 football teachers; there are 1 national level athletes, accounting for only $4 \%$ of all teachers; 3 of them are national level 2 athletes, accounting for $11 \%$ of all teachers. There are 7 national level 3 athletes, accounting for $22 \%$ of all teachers. There are also quite a few teachers who do not have a football player rating, which accounts for $63 \%$ of all teachers, a high percentage. Therefore, the vast majority of football teachers have not experienced football professional training, have no experience of playing in professional teams, and are relatively weak in football practice, which will affect the quality of these teachers in daily training guidance and competition command. After investigation, some football teachers received a professional soccer training starting point rather late. They started from high school or even university. Their own level of skill in football matches that of football players is relatively low, so the level of student football developed by students is naturally not too high.

The Investigation and Analysis of the Referee's Grade of Football Teachers in Each Pilot School. Among the pilots of the campus soccer school, there is no national referee-level football teacher, one is a national first-rate referee, two are national second-tier referees, five are national three-level referees, and there are 22 football left The teacher does not have a referee level, which means that teachers who do not have a referee level are more than double the total number of football teachers who have a referee level. A formal football match must be equipped with a qualified referee. The Nantong City Campus Football League has a total of 30 teams. It adopts a double-loop system of home and away games. The amount of referee work is large, so a large number of referees are required. However, many football teachers currently training football for teenagers are lacking experience as referees and have not conducted relevant referee training. Therefore, these factors have also constrained the popularization of campus football activities in Chibi.

Survey and Analysis of Training Situation of Football Teachers in Various Pilot Schools. The "National Youth Campus Soccer Activity Implementation Plan" requires football teachers in pilot schools to provide relevant training for football coaches. In coaching training, the training ability of football teachers has an important influence on the improvement of football players' skills and tactics. According to the survey, 30 football teachers from each pilot school participated in the football coach training course. The training course for football teachers set up two parts: practical content and theoretical content. During the course of knowledge learning, we conscientiously implemented the 
requirements of the Hubei Football Association. We have specialized staff to arrange the daily operation of the training courses, register attendance rates for 30 teachers, and whether there are any late and absent records. The training lecturer teaches on the basis of the teaching content and the football teacher listens to the records carefully. , discuss topics in groups. The practical lesson is demonstrated by training instructors. The teachers actively cooperate with each other to complete the tasks in groups and finally complete the assessment in both theory and practice. The training content of this training course is mainly divided into: 1. The physical and psychological development characteristics of primary and secondary schools. 2, coach instructors work content and obligations. 3 , training content and training methods. 4, organizational measures and safety precautions. 5, football festival process and design football section. After two days of outdoor practice, the teachers made it clear how they should arrange the contents of the training course and the essentials of each technique and tactics. Clearly understand the responsibility of the football teacher, master the teaching methods of football basic skills and tactics, and practice methods for athletes' physical fitness. In particular, it is clear how to give a certain class of students a good training class, how to guide teamwork, and how to lead a team. After everyone's efforts, the training course finally closed successfully. After this activity, each football teacher clarified the necessity and arduousness of carrying out the campus soccer campaign, defined his mission, and laid a solid foundation for the smooth promotion and popularization of school football in the future.

Each school's football teacher has worked hard to learn, practice, rationally combine theory and practice, absorb advanced knowledge, and receive scientific ideas so that he can become an outstanding football coach on the day of training and competition command. We are subtly improved.

\section{A Case Study of Chibi 1 and Chibi 2}

A Case Study of the Development of Campus Football in Chibi Primary and Middle Schools. The Chibi 1 Chinese football team is a traditional strong team in Chibi. The team's predecessor was in the middle of Chibi, and later renamed it as a middle school. From the beginning of the school competition, students with outstanding performance were recruited into the school team, combined with the recruitment of football talents. Science system training that maintains a regular pattern throughout the year. He often participates in many major competitions in provinces and cities. According to the purpose of the "Cultural Classes combined with football training", the school sets off a good atmosphere of competition, so that players realize that: To become a high-level football player is inseparable from the accumulation of culture. To cultivate the player's sense of responsibility and collective consciousness, actively shape the player's good psychological quality, attitude and perseverance, and enhance their football skills and execution ability. Since the school team was established, it has been the champion of the high school team in the Chibi Campus Football League. It can be said that the level of football in Chibi represents the highest level in the same age stage in Chibi.

According to the status quo, Chibi One has developed soccer training textbooks in a targeted manner. The school began with scientific and reasonable module training, and at the end of the period there will be assessments of training results. Football has become a must to study in physical education classes. Each student will have a football course to study for a week. The teacher will systematically introduce the history of world football development to the children in class and let students scientifically and rationally learn basic skills such as kicking, dribbling, passing, stopping and shooting. In order to stimulate students' perseverance and hard work, they cultivate the idea of time, the collectivism and teamwork spirit of "one for everyone, and one for everyone" and cultivate good social adaptability. In addition, there is a football training class at each weekend in City 2. The school hires high-level professional coaching coaches to provide students with a good playing environment as much as possible. Let more students come into contact with this sport and participate in this activity so that their mind and body can be fully developed.

A Case Study of the Development of Football Activities in Chibi Second Campus. Since Chibi started small school two years ago, it has been paying attention to students' bodies and bodies. The 
"Opinions on Strengthening the Juvenile Sports System to Strengthen the Juveniles" promulgated by the State has been implemented, and the promotion of the campus football culture has been deepened. The basic skills of students have been improved, training content has been enriched, and innovative ideas have been incorporated into practical lessons. Due to the increasing number of provincial-level provincial youth competitions, the younger players in the Chibi two have been getting more and more performances on their own stage. Chibi had participated in the city's competition for many years and achieved outstanding record: he has won several championships in the city's primary school. Schools will also actively adopt incentives to motivate students. For more than a decade, Chibi Erxiao has always put children's sports work in an important position. According to the characteristics of the size of the school's event venues, the world's first sport -------- football sports, to create football characteristics.

How can students who rarely touch the ball recognize football, enjoy football, and become interested in football? School

More time for the students to get in touch with football, the provision of physical education must have some time for football teaching, and extra-curricular activities are also focused on the arrangement of football content. As a result, students never touch the ball to touch the ball and gradually touch the ball. Love to play, many classes have also set up a small football team. The school took full advantage of this opportunity and began the second step. It was to establish the "three cups" league in the school, namely: "Miao Miao Cup", "Winning Cup” and “Champion Cup”. In third grade or above, each year of the semester takes the class as the unit, to perform the grade football round-robin. Seeing that the students' feelings for football is deeper, there are more students practicing the ball, and there are more students who dare to fight hard on the field. The "three cups" league led to the formation of football characteristics. The third step is to make full use of external forces to promote the rapid development of football. The school and the football club jointly organized the Chibi Children's Soccer Base and added some necessary facilities such as football walls and nets. The popularity of school football is getting higher and higher, and even some girls are now actively involved and they form their own teams. While popularizing football, the school also attaches great importance to the training of the "Miao $\mathrm{Zi}$ " team members and strives to provide excellent reserve talents for teams at all levels. Made a contribution to China's football career.

Football sports have promoted the overall development of sports. Through exercise, students mastered certain sports skills, enhanced their physical fitness and ability to resist diseases, and also cultivated students' aggressive spirits of not being afraid of suffering, not being tired, not afraid of setbacks, and being courageous to fight.

\section{Constraint Factors of Campus Football Activity in Primary and Middle Schools in Chibi City}

School Leaders Don't Pay Enough Attention. A small number of pilot school leaders pay less attention to the development of youth football, and they have the idea of "emphasizing the importance of evasiveness." Schools only pay attention to the academic class's academic performance, do not attach importance to the overall development of students' mind and body, and combat young people's passion for football. As a superficial phenomenon, it is considered that the primary and secondary school campus soccer activities are simply to participate in a few games, and that the students do not guarantee that at least one football lesson a week and 120 minutes of football time are stipulated. In football, there is no football match between the grade level and the entire school. The reason for the unsatisfactory results of the development of the youth campus football in some pilot schools is that school leaders do not pay attention. Even some schools have temporary teams. Only a very small number of pilot schools have a certain level of competition, but they are generally considered horizontal. Still uneven.

The Source of Funds for Campus Football Activities is not Guaranteed. As a long-term project, the development of campus football activities in primary and secondary schools requires a fund-based guarantee. countryThe General Administration of Sport allocates RMB 40 million each year from the sports lottery public welfare fund to provide a strong guarantee for the school to carry out campus soccer, and it is the main funding for the development of youngsters' campus 
football. However, relying solely on funds from national, provincial and municipal sports departments at various levels cannot solve the fundamental problems. And companies and social groups are seldom willing to sponsor and think there is no return without influence. Constraints on the development of campus football in primary and secondary schools in Nantong also include the lack of multi-channel development of market resources. Therefore, the education department must provide a certain amount of financial support, provide funds on the basis of a ratio of not less than 1:1, and ensure the smooth development of the youth campus soccer game.

Unbalanced Football Activities on Campus. The promotion and popularization of school football in primary and secondary schools in Chibi City presents a serious imbalance. Among them, the traditional football schools are ahead of other pilot schools, not only in the performance of the campus league, but also in the positive attitude of the school leaders and the cooperation of parents. Supporting and popularizing the youngsters' campus football culture, and carrying out campus football activities according to the requirements of the "National Youth Campus Soccer Activity Implementation Plan" require more perfection than the late-stage school football pilot schools. Schools that start late on campus football can observe the schools that started early on in campus football activities and learn from their good experiences. Schools that have experience in carrying out campus football should also provide support and assistance to schools that have started late. Only under such a healthy circulation environment can Chibi campus football in primary and secondary schools be more sustainable and benign.

The Professional Quality of Football Coaches is not High. The Chinese Football Association has not been adequately trained in the training of coaches at the professional level. As a result, there is a situation in which the lack of high-level coaches in the country has occurred. Moreover, the number of young coaches is low and the number is not enough. Despite the use of domestic primary and secondary school campus football as a route, the Football Association has organized many lecturer training courses and D-level coach training, but these cannot fundamentally solve the problem of low professional quality of domestic young coaches. This is an important factor affecting the development of youth sports on campus. There are very few teachers with professional football backgrounds in the schools that carry out youth sports on campus because many schools do not recruit teachers with professional football skills. Many schools can only allow teachers who have not been contacted by football to lead training games. The trained teenagers will not have much improvement in football skills and tactics, and even waste valuable time. The level of professionalism of the football teacher can show the training level of the players and the ability to adjust and control the game during the game. At present, many teachers who conduct campus football have not received systematic and professional football studies and have not received training from football coaches. As a result, the training content of these teachers has been arranged, and the arrangement of games has been lacking in scientific and rational arrangements. There are many problems in the demonstration and explanation. Other obstacles to the development of campus football in the elementary and secondary schools in Nantong include football coaches' level of coaches, umpires, academic qualifications, and athletes' ranks.

A Considerable Number of Educational Institutions are Perfunctory. Although all 30 experimental schools in Chibi have joined the city's campus football team, students are selected according to the criteria to form a football team for training and competitions. However, fewer schools actively promote school football on primary and secondary schools. Many schools are in fact mass-producing, and they simply do not pay attention and perfunctory. Among these schools, some schools believe that as long as there is a stadium, a team, and a student who can play football on the court, they do not care how many students participate, what level they can kick the ball into, and what the score is in the league. Do not pay attention, even if the students have kicked the ball on the venue. Ignoring the primary and secondary school campus football is to bring as many students as possible into the sport of football, inspire young people to have an interest in football and gain happiness from football. Young people can acquire the theoretical knowledge of football from the participation in the primary and middle school campus football, master the technical skills of football, and create a youthful campus football culture atmosphere, so that young people can't live without the glamorous 
"world's number one sport" of football. In order to further educate the body and mind to develop a comprehensive, large-scale future of Chinese football.

Insufficient Cooperation with Sports and Education Departments. Juvenile campus football is a project jointly developed by the Ministry of Education and the State General Administration of Sport, and it is a joint and difficult task. To implement the primary and secondary school campus football, each school under the Ministry of Education is dominant. Judging from the situation of the implementation of campus football in primary and secondary schools in Nantong City, there are few people in the education sector who can really work hard for this work. The education sector lacks specific recommendations and guidance for the primary and secondary school campus football. The activity of primary and middle school campus football is the best embodiment of "combination of physical education and education", and it is an important way to cultivate students of both civil and military. We should let the education department and the sports department cooperate with each other and work in close cooperation to create a very strong atmosphere for the promotion and popularization of school football in primary and secondary schools. Only in this way can we promote the overall popularization of school football in primary and secondary schools. However, in view of the still-exploration period, there are still some problems in the process of the development of youngsters' football on campus, which is reflected in the "combination of sports and education". The excessive interdependence between the sports department and the education department has resulted in an incomplete system of activities; the activities of the organization are still unreasonable; the competition plan is not scientific; the responsible managers of the competition are not responsible enough; the audit work is not enough to be done. "Combination of sports and education" should not only be spoken verbally, but must be truly reflected in the work of campus football. The degree of close cooperation between the education department and the sports department is not enough to hinder the implementation of the youth campus soccer strategy in certain aspects. Therefore, the sports department and the education department should shorten the cognitive distance of young people's campus football. The development of the youth campus football needs not only to select talented students to become football reserve talents, but also to make football the "world's number one sport". Students who can become lifelong benefits for the students and educate students who are fully developed and physically and mentally healthy. The two complement each other.

\section{The Development Strategy of Youth Campus Football in Chibi}

Strengthen the Importance of Leadership and Ensure that Ideas are in Place. School leaders need to change their own ideas, systematically conduct football training for young people, and scientifically arrange the intensity of youth sports. This not only does not delay the students' academic work, but also cultivates young people's interest in knowledge, all positive things, and promotes the health of young people. The degree of attention of the leaders is an important factor in promoting the popularity of school football. The relevant leaders of each pilot school must be fully aware of the fact that they must pay close attention to school football and provide support in all areas to spend money on the place where they spend. In the renovation of the stadium, teachers, equipment purchases, participation in competitions and other places need personal guidance and inspections, so that to a certain extent, they provide very important support to the main teachers in charge of campus football activities. The leaders of each pilot school must understand the connotation of school football and master the relevant standards for the "National Youth Campus Soccer Activity Implementation Plan”. In this way, school football can be truly popularized.

Unify Thoughts and Actively Create a Campus Football Culture Atmosphere. By building a good school football culture atmosphere, we will increase the youth's initiative and interest in participating in football. Therefore, each experimental campus should pay attention to the construction of school football culture, such as increasing the promotion of school football culture, changing the sports competition and the after-school physical training model, enriching the content of football learning, enriching the contact methods of youth school football culture, and holding various Various kinds of school football culture movements, designing a scientific and reasonable 
propaganda system for school football culture, perfecting football team formation, formulating campus football cultural propaganda programs, carrying out more student football practice classes, and holding "school football cultural festival" and other activities.

Further Establish and Improve the "Students and Teachers Combined" Football Reserve Personnel Training System. It is a difficult task to carry out school football in primary and secondary schools. It requires that the two departments of the teaching body closely cooperate with each other to do a good job in this work and strive to integrate football into schools. Campus football activities provide schools with opportunities for "combination of teaching and learning". Through the establishment of systematic and detailed regulations on "combination of teaching and learning", the exchanges between the sports bureau and the Ministry of Education are increased, the tasks are clearly distinguished, and the conditions are used. With its advantages, it actively provides all aspects of resources needed for the development of campus soccer, provides a good environment, and gives a solid foundation for the implementation of school football. This will enable school football to be successfully carried out. In the development of football reserves, we combine the advantages of education and sports to achieve each other's strengths and weaknesses, courageously assume responsibility and obligations, and share results. However, at the initial stage, the Education and Sports Bureau must demonstrate determination, introduce advanced ideas, regularly study the problems that arise, overcome difficulties faced, draw valuable experience, and actively explore the research direction, advanced methods, and advanced systems of "combination of teaching and learning”. Step by step to improve the favorable model for carrying out school football activities.

Strengthen the Propaganda Work of Campus Football Activities and Effectively Promote the Development of Campus Football Activities. The organization of school football is inseparable from the support of people from all walks of life. Therefore, the task of increasing the promotion and popularization of school football is also a key link in the implementation of school football in the future. Each pilot campus should use the campus network, school broadcasting resources, and use corridors to paste football knowledge to popularize school football, highlighting the tremendous changes that school football has brought to schools. In school, the idea of "happy football, sunshine sports" is reflected in the way that physical education courses are taught, various kinds of sports are held, and active competitions are registered. In addition, each experimental campus should actively use the Internet platform to increase the content of "school football" on the Internet. Everyone has the right to browse, so that more and more teachers and parents join together, and regularly update the content and publish. The students' training experience, the combination of text and pictures, and the publication of various photos of the pilot school football competition and the school team training, promoted the introduction of the status quo of the development of the campus football, so that the school football is not just a sport for the players to participate in. The sport will also affect the family members of the players as well as all relatives and friends and people in society. The promotion and popularization of school football concepts and sports is crucial to the development of school football. The Chibi Bureau of Education must first complete the design of the school football website in Chibi, and combine it with each press and propaganda department in Chibi, through newsletters, introductions, special topics, photos, interviews, and other promotional channels. Sexual introduction of the implementation of football in primary and secondary school schools in Chibi was introduced to the public and eventually reached the goal of truly promoting school football.

Government Agencies should always Organize Pilot School Football Teachers to Carry out Football Training. Government agencies should always organize pilot school football teachers to carry out football training, carry out work seminars and summaries among coaches, exchange experiences, increase purposeful and targeted coaches' knowledge reserves and improve practical ability, and improve coaches' professional concepts. With special skills technology. Then, each pilot's campus will invite football professionals and professional team players, as well as team coaches who will lead the team on the first day to come to the campus for coaching and demonstration. This will also help deepen the responsibility for carrying out campus football. The coaches' understanding of this activity will improve the quality of the football teacher's actions, learn from each other's strengths, and innovate the training content of football teachers so as to enhance the 
professional qualities of the coaches. In addition, it is necessary to increase the salary standards of coaches, give them various material resources needed for the development of campus football activities, and better stimulate the enthusiasm of teachers to lead training and competitions and reflect their own values. Of course, it is necessary to establish an assessment mechanism and conduct regular business evaluations on teachers. There is a certain incentive policy for football teachers with outstanding scores, which not only plays a role in motivating but also improves the quality of football teaching work for teachers.

Strengthening the Supply of Government Sports Public Services. The extensive development of youth football is included in the development planning of government departments. The municipalities shall build a non-academic football activity center with no less than three natural turf in the urban green space. The sports lottery public welfare fund shall be subsidized. The organization shall be open to young students for free within the prescribed time; the cities that completed the "new four ones” project (county), Area)

The center's sports venues are free to organize and open to young students within the specified time and expand the youth football school.

The number of external activity outlets and the number of people who regularly participate in off-campus football activities.

Encourage social forces to help youth football. We will guide social forces to actively establish youth soccer football clubs and other football outreach organizations, youth football series, and youth football series. Support schools and joint social forces organizations to carry out weekend training camps.

Improve Guidance Policies and Incentives for Further Studies. Improve policies for enrollment from primary, middle and high schools to encourage young people to participate actively in the long term.

Football learning and training. We will improve the enrollment policy for high-level college football teams and expand the scale of enrollment.

The student's football learning, training, competition, and football skill level are included in the student's comprehensive quality assessment file as a basis for students' entrance examinations and award evaluation.

Establish and Improve the Safety Guarantee System for Youth Football Activities. Establish and improve a youth football risk management system covering safety education and training, activity process management and insurance compensation. Establish an emergency management mechanism for accidental injuries on campus football. Strengthen the management and maintenance of football venues and facilities to eliminate safety hazards in time.

\section{Conclusion}

In the Chibi School Soccer School for Primary and Secondary Schools in Chibi, 33\% of the targeted school football leadership institutions have not been established according to the "National Youth Campus Soccer Activity Implementation Plan"; 46.7\% of all school football matches have been held; The time of $2 \mathrm{~h}$ reached $17 \%$; only $13 \%$ of the students participated in football when the number of students reached more than half. The vast majority of campuses are limited to the training of the school team and participate in the campus football league. The slogan of "All people participate in and actively promote" the school football game has not been implemented. There is a small number of pilot campus activities that go through the field.

There is not enough enthusiasm for soccer culture propaganda in primary and secondary schools in Chibi. Only about 37\% of schools have fully promoted and promoted the school's football culture. Other pilot schools did not pay much attention to the campus football cultural propaganda work, which to some extent hindered the development of school football and the enthusiasm of young people to participate in football. 
The education level of soccer teachers in pilot schools in Chibi is mainly undergraduate. $37 \%$ of the teachers have the level of football players, $27 \%$ of the teachers have the rank of referees, most teachers have low special skills, and the relevant professional qualities urgently need to be improved.

The conditions of the hardware (football field) of the experimental schools in Chibi primary and secondary schools are generally satisfactory. Overall, they can ensure daily training and youth football. In addition, some schools' stadiums are not up to standard, and 7\% of the school's courts are soil. Impede the smooth development of school football.

The progress of the development of campus football in primary and secondary schools in Chibi is not uniform. Early starting schools had a good foundation for development and the atmosphere of football was relatively strong. In schools that started late, the lack of equipment, professional football teachers, and school principals were not enough attention. Therefore, when it comes to school football, there is not a small gap with the traditional football school.

The coordination between the Ministry of Education and the Bureau of Sports of Chibi on the supervision of the league, the hosting and management of the competition, the cost of the competition and the training of the football teacher are not enough; the person in charge of a few activities does not have a thorough understanding of the national campus football activities and needs improvement. Work attitude.

\section{Acknowledgement}

This research was financially supported by the National Science Foundation.

\section{References}

[1] Li Jixia, Dong Zhongming, Xu Yangcai et al. Research on the Management System Innovation of Teenagers' Campus Football in China[J]. Journal of Shandong Institute of Physical Education, 2012, 28(3): 99-104.

[2] Guan Li, Zhang Hongjia. Investigation and Analysis of Family Factors Influencing the Popularization of Youth Football[J]. Journal of Shenyang Physical Education Institute, 2005(4):26.

[3] Liu Bing. Present situation and development countermeasures of our country's school football amateur training[J].Journal of Anhui Sports Science,2002(2):4.

[4] Liao Yanwang. The current situation and countermeasures of the development of urban middle school football in Hunan[D]. Chongqing: Southwest University, 2009:16.

[5] Zhou Zhicheng. Investigation on the status quo of women's football in primary and secondary schools in Wuhan[D]. Hubei: Huazhong Normal University

University, 2006:9.

[6] Liu Weimin. Analysis and Countermeasures of the Main Factors Affecting the Development of Youth Football in Wuhan[J]. Journal of Hubei Normal University. 2009(2): 67.

[7] Liu Jia. Some problems and countermeasures for the training of football reserve talents in Zhejiang Province [D]. Zhejiang: Zhejiang Normal University, 2009: 5.

[8] Wang Depeng. China's football from the top three leagues in China to see the Chinese football league mechanism [D]. Jilin: Jilin University, 2008:20.

[9] Sun Yi, Liang Yongqiao, Bi Haibo. A Comparative Study of Junior Football Training Systems in China, Japan and South Korea[J]. China Sport Science and Technology, 2008(4):376.

[10] Nie Xiaohu. The important move of German football reform [J]. Sports Culture Guide, 2003, $1: 55-56$. 
[11] Guo Jingtao. A Comparative Study on the Training Model of Youth Football between China and Germany[D]. Shandong: Shandong Normal University, 2011:11.

[12] Guo Jingtao. A comparative study on the talent training model for youth football in China and Germany [D]. Shandong: Shandong Normal University

Journal of 2011:7.

[13] Sun Kecheng, He Zhilin, Dong Zhongming. The Training Path and Enlightenment of Foreign Reserve Talents for Soccer Powers[J]. Journal of Nanjing Institute of Physical Education, 2011(5):234-237. 